\title{
Partially Quenched QCD with Non-Degenerate Dynamical Quarks *
}

\author{
S. Sharpe ${ }^{a}$ and N. Shoresh ${ }^{a \dagger}$

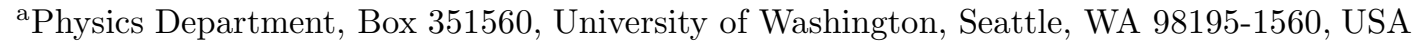

We discuss the importance of using partially quenched theories with three degenerate quarks for extrapolating to $\mathrm{QCD}$, and present some relevant results from chiral perturbation theory.

Simulations of lattice QCD have, to date, largely used the quenched (Q), or partially quenched (PQ), approximations. These approximations introduce unphysical artifacts, some of which can be analyzed using chiral perturbation theory (ChPT) [1 3 . In particular, it has been found that the quenched theory has unphysical singularities in the chiral limit, and that these persist in the PQ theory, albeit in weakened form [3 5 . These results serve as a warning against relying on $\mathrm{ChPT}$ when straying too far from unquenched theories in which valence and dynamical masses coincide.

In this talk we discuss a more constructive aspect of partially quenched QCD. We focus on PQ theories with three light dynamical quarks, where "light" means that the theory can be studied using ChPT. Such theories have the same quark complement as QCD, although with differing masses. When simulating such theories it is possible to push to much lighter valence quarks than dynamical quarks, and thus to map out a "rectangular" region in the space of theories as sketched in Fig. 1. Our aim is to make use of the extra information contained in the PQ data to learn about unquenched theories, and in particular about real QCD. The key point is that, as long as one stays far enough away from the $m_{V}=0$ axis, ChPT reliably predicts the functional dependence on valence and dynamical quark masses, and so provides a unified description of both the PQ and fully unquenched regions of the parameter space.

An observation central to this work is that the

\footnotetext{
*Supported by DOE contract DE-FG03-96ER40956.

${ }^{\dagger}$ Speaker
}

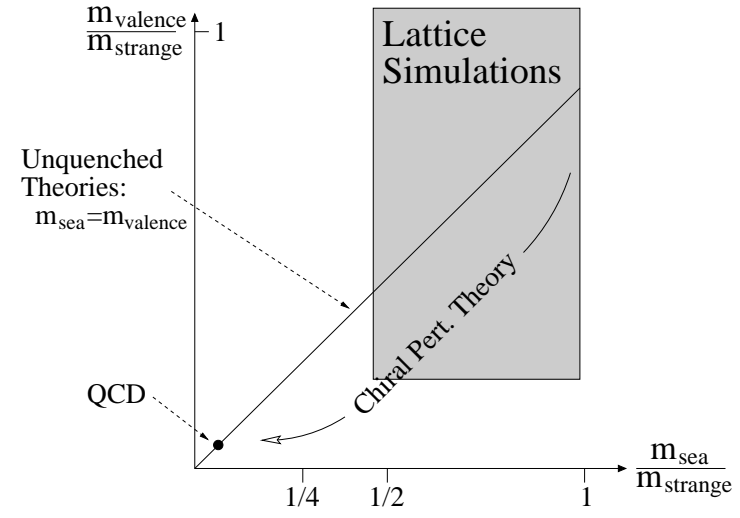

Figure 1. Schematic representation of the space of PQ theories.

unknown coefficients appearing in the chiral Lagrangian for PQ theories depend only on the number of light dynamical quarks, and not on their masses [4]. One way of viewing the extra input that $\mathrm{PQ}$ theories provide is that they allow a cleaner separation of the non-leading, $O\left(p^{4}\right)$ coefficients. This is because some GL coefficients appear multiplied only by valence masses, others only by dynamical masses (see Eq. (2)).

The picture that emerges is that, by simulating in a region of moderately light valence and dynamical quarks, and varying independently the masses of both, one can determine all the coefficients necessary to extrapolate to QCD. This viewpoint has also been stressed in Ref. [6] in the context of determining the up quark mass. The resulting accuracy is limited by the order to which one calculates in ChPT.

\footnotetext{
${ }^{3}$ This result is also implicit in the work of Ref. [3].

${ }^{4}$ Which we refer to as Gasser-Leutwyler (GL) coefficients.
} 
An important question is whether it is necessary in such a program of calculations to consider non-degenerate dynamical quarks. For the quantities we consider here - pion masses and decay constants - degenerate dynamical quarks suffice at one-loop in ChPT, because the expressions contain only the sum of the dynamical masses 5 . Simulations with non-degenerate dynamical quarks are necessary for more accurate chiral extrapolations, since mass differences enter in two-loop ChPT results. Such simulations are also attractive because they bring one closer to QCD, and thus reduce the dependence on ChPT. They may also be advantageous from a computational point of view - since a dynamical strange quark is relatively cheap, one may approach more closely to QCD by having two lighter dynamical quarks than three.

For these reasons we think it likely that simulations with non-degenerate dynamical quarks will be done, and thus as a first step in our program we have calculated the one-loop expressions for the masses and decay constants of the pions and kaons in a theory with three dynamical quarks, only two of which are degenerate. This generalizes Refs. [4,5], which calculated these quantities with any number of degenerate dynamical quarks.

The partially quenched approximation can be described in terms of a Lagrangian by adding a number of commuting spin- $1 / 2$ (ghost) fields [7]. For each valence quark there is a corresponding ghost quark, and when their masses are equal the valence quark loops cancel against their ghost counterparts. The dynamical quarks, which do not have corresponding ghosts, give rise to the quark loops that are kept in the simulation. The complete mass matrix is

$M=\operatorname{diag}\left(m_{V 1}, m_{V 2} ; m_{S 1}, m_{S 2}, m_{S 3} ; \widetilde{m_{V 1}}, \widetilde{m_{V 2}}\right)$,

where $V$ refers to valence, $S$ to sea or dynamical, and a tilde indicates ghost quantities. In our theory $m_{V i}=\widetilde{m_{V i}}$, and $m_{S 1}=m_{S 2}$. For baryons one would need to include an additional valence quark and its corresponding ghost.

The approximate $\mathrm{SU}_{\mathrm{L}}(3) \otimes \mathrm{SU}_{\mathrm{R}}(3)$ chiral symmetry of QCD generalizes to the graded group

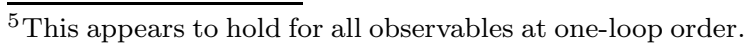

$\mathrm{SU}_{\mathrm{L}}(5 \mid 2) \otimes \mathrm{SU}_{\mathrm{R}}(5 \mid 2)$ in the PQ theory $[3]$. Assuming that this larger symmetry is broken spontaneously down to its vector subgroup, one finds a large multiplet of pseudo-Goldstone particles which includes both bosons (quark-antiquark and ghost-antighost) and fermions (quark-antighost). Collecting these into a matrix, $\Phi$, and defining $\Sigma=\exp (2 i \Phi / \mathrm{f})$ and $\chi=2 \mu M$, the chiral Lagrangian describing the low-momentum interactions of these particles is [3, 4]

$$
\begin{aligned}
\mathcal{L}= & \left(\mathrm{f}^{2} / 4\right) \operatorname{str}\left(\partial \Sigma \partial \Sigma^{\dagger}\right)-\left(\mathrm{f}^{2} / 4\right) \operatorname{str}\left(\chi \Sigma^{\dagger}+\Sigma \chi\right) \\
& +\alpha_{\Phi} \frac{1}{2}\left(\partial \Phi_{0}\right)^{2}+\frac{1}{2} m_{0}^{2} \Phi_{0}^{2} \\
& +L_{4} \operatorname{str}\left(\partial \Sigma \partial \Sigma^{\dagger}\right) \operatorname{str}\left(\chi \Sigma^{\dagger}+\Sigma \chi\right) \\
& +L_{5} \operatorname{str}\left(\left(\partial \Sigma \partial \Sigma^{\dagger}\right)\left(\chi \Sigma^{\dagger}+\Sigma \chi\right)\right) \\
& -L_{6} \operatorname{str}\left(\left(\chi \Sigma^{\dagger}+\Sigma \chi\right)^{2}\right) \\
& -L_{8} \operatorname{str}\left(\chi \Sigma^{\dagger} \chi \Sigma^{\dagger}+\Sigma \chi \Sigma \chi\right)+\ldots
\end{aligned}
$$

This form is the same as that for QCD except for the replacement of traces by supertraces, and for the appearance of the super $-\eta^{\prime}$ field $\Phi_{0} \propto \operatorname{str}(\Phi)$. As in standard ChPT there are unknown constants at each order: f, $\mu, \alpha_{\Phi}$ and $m_{0}$ at leading order, and the GL coefficients $L_{i}$ at one-loop (of which only those that are relevant are displayed). Each term in Eq. (11) can also be multiplied by an arbitrary even function of $\Phi_{0}$. These additional functions do not, however, enter into our final results and are not shown.

The QCD chiral Lagrangian is completely contained in Eq. (11), and can be obtained from it by setting valence and dynamical quark masses equal [3]. Moreover, since all quark mass dependence appears explicitly in Eq. (1), and all the unknown coefficients are independent of masses, these coefficients are the same for the partially quenched theory and full QCD.

Using Eq. (1), we calculate the masses and decay constants of the Goldstone particles to one-loop in ChPT [8]. The results are functions of valence and dynamical masses: $\quad M^{2}\left(m_{V 1}, m_{V 2} ; m_{S 1}, m_{S 3}\right)$ and $F\left(m_{V 1}, m_{V 2} ; m_{S 1}, m_{S 3}\right)$. Unquenched pion-like properties are obtained for $m_{V 1}=m_{V 2}=m_{S 1}$, whereas kaon-like properties are obtained for $m_{V 1}=m_{S 2}$ and $m_{V 2}=m_{S 3}$. The results contain 
a simple term analytic in quark masses, e.g.

$$
\begin{aligned}
M^{2}= & \mu\left(m_{V 1}+m_{V 2}\right)-\left(8 \mu^{2} / f^{2}\right)[ \\
& 2\left(L_{4}-2 L_{6}\right)\left(m_{V 1}+m_{V 2}\right)\left(2 m_{S 1}+m_{S 3}\right) \\
& \left.+\left(L_{5}-2 L_{8}\right)\left(m_{V 1}+m_{V 2}\right)^{2}\right],
\end{aligned}
$$

and a long and unilluminating chiral logarithm arising from loops. Note that the dependence of $M^{2}$ on $\left(m_{V 1}+m_{V 2}\right)^{2}$ gives the combination $L_{5}-$ $2 L_{8}$, the value of which is needed to determine the physical $m_{u p}[6]$.

We use the full one-loop expressions to study issues that arise in the extrapolation towards QCD. To do this we use a simplification noted in Ref. [4]: for three dynamical quarks, the super- $\eta^{\prime}$ has a mass comparable to the physical $\eta^{\prime}$ and can be integrated out. The resulting forms are independent of the parameters $\alpha_{\Phi}$ and $m_{0}$ and the extra functions of $\Phi_{0}$ implicit in Eq. (11). They thus depend only on parameters that are present in the QCD chiral Lagrangian. We then find values for $\mathrm{f}$, and for the GL coefficients, such that there are choices for the "physical" up and strange quark masses, denoted $m_{u p}$ and $m_{s t}$, which reproduce correctly the experimental values of $f_{\pi}$, $f_{K}, m_{\pi}$ and $m_{K}$. There is a range of allowed GL coefficients - we select representative samples by the additional criterion that chiral perturbation theory should be converging.

Our first study concerns the importance of including non-analytic terms when extrapolating to QCD. We produce "fake" data by calculating $M^{2}$ and $F$ (using the full one-loop forms) on a preset grid of quark masses. The data is then fit to the one-loop analytic form predicted by ChPT (e.g. Eq. (22)), excluding the chiral logarithms. This fit is then used to extrapolate to the physical values for the ratios $m_{\pi} / f_{K}$ and $m_{K} / f_{K}$. This leaves $f_{\pi} / f_{K}$ as a "prediction", which can be compared with the exact value. We find that, for grids with masses above $\sim m_{s t} / 8$, this procedure leads to $10-15 \%$ errors in $f_{\pi} / f_{K}$. This is true also if one extrapolates solely using unquenched data. We conclude that the extra curvature due to the chiral logarithms is substantial, and needs to be incorporated in the extrapolation.

Most current PQ simulations use two dynamical quarks, which can be viewed as the infinite

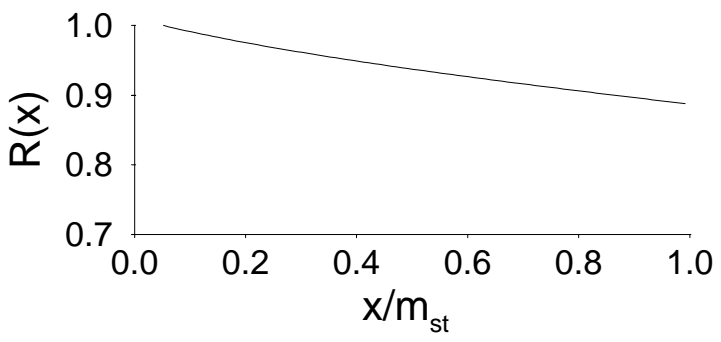

Figure 2. Effect of varying the dynamical strange quark mass. Quark masses are $m_{V 1}=m_{u p}$, $m_{V 2}=m_{s t}, m_{S 1}=m_{S 2}=m_{u p}$ and $m_{S 3}=x$. $R(x)=\left[M_{K}(x) / f_{K}(x)\right] /\left[M_{K}\left(m_{\text {up }}\right) / f_{K}\left(m_{\text {up }}\right)\right]$.

strange quark mass limit of the three flavor theory. An important issue is how large an error one makes by excluding the dynamical strange quark. ChPT cannot be used to calculate this effect, since it cannot be applied if $m_{s t}$ is too heavy. Nevertheless, by varying $m_{s t}$ within the range of validity of ChPT, one can obtain an estimate of the error. Fig. 2 2 shows how the (normalized) ratio of $m_{K} / f_{K}$ varies as the mass of the third dynamical quark is increased from $m_{u p}$ (i.e. three degenerate dynamical quarks) to $m_{s t}$ (the physical point). Note that the valence quark masses are unchanged. We see an effect of $\sim 10 \%$-large enough to suggest that inclusion of the dynamical strange quark is important.

More details concerning these exercises, as well as the one-loop results for three non-degenerate quarks of arbitrary mass, will be given in [8].

\section{REFERENCES}

1. C.W. Bernard and M.F.L. Golterman, Phys. Rev. D46, 853 (1992).

2. S. Sharpe, Phys. Rev. D46, 3146 (1992).

3. C.W. Bernard and M.F.L. Golterman, Phys. Rev. D49, 486 (1994).

4. S. Sharpe, Phys. Rev. D56, 7052 (1997).

5. M.F.L. Golterman and K.-C. Leung, Phys. Rev. D57, 5703 (1998).

6. A. Cohen, D. Kaplan and A. Nelson, hep-lat/9909091.

7. A. Morel, J. Phys. (Paris) 48, 1111 (1987).

8. S. Sharpe and N. Shoresh, in preparation. 